Military Technical College Kobry El-Kobbah, Cairo, Egypt

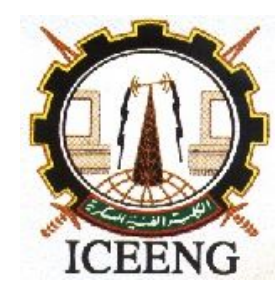

\author{
$10^{\text {th }}$ International Conference \\ on Electrical Engineering \\ ICEENG 2016
}

\title{
A Microcontroller-Based Heart Sounds and Murmurs Generator Using Discrete Wavelet Transform
}

\author{
By
}

M. E. Karar*

\section{$\underline{\text { Abstract: }}$}

The most important problem of biomedical engineering either for teaching or research activities is how to obtain data of patients with different clinical situations, because it is difficult to find unhealthy volunteers and also to get ethical approval for any experimental test on patients. Hence, this paper presents a practical generator of heart sounds and murmurs for educational and research purposes. The developed generator produces graphical representations of normal and two abnormal heart sounds, which are aortic stenosis and mitral insufficient. Three datasets of real heart sounds have been preprocessed using discrete wavelet transform, in order to reduce the noise and decompose these cardiac signals to the third approximated level based on Daubechies four-coefficient wavelet. For designation and implementation of the heart sounds generator, the ATmega 2560 microcontroller was carefully chosen to produce the output of all preprocessed datasets. The developed microcontroller-based generator showed successfully different cases of the heart sounds and murmurs, allowing to be used further for testing new automatic classification methods of cardiac valve diseases.

\section{Keywords:}

Phonocardiography, heart signals simulation, wavelets, Arduino microcontroller board

* Faculty of Electronic Engineering (FEE), Menoufia University 


\section{Introduction:}

\section{A. Medical Background}

Heart sounds are mainly associated with the electromechanical activity of the cardiac muscle, including the systole and diastole of the atria and ventricles, and the closure and opening events of the valves during each heart beat [1]. Cardiac auscultation is the process of interpreting the heart sounds [2]. Therefore, it can be used for the early detection of abnormal function of the heart before carrying out any further diagnosis procedures.

Stethoscopes are usually used to acquire and hear the heart sounds. However, the physician needs extensive training and experience with good hearing ability to perform effectively the auscultation technique. In addition, advanced electronic stethoscopes are sometimes required to make clearly all portions of the heart signals audible. Alternatively, the phonocardiography displays the sounds produced by the heart in a graphical form as similar as the electrocardiogram (ECG) [3]. Hence, any cardiac abnormality can be visually appeared in the corresponding phonocardiogram (PCG) signal.

The first and second heart sounds are two dominant and loudest sounds; namely S1 and S2 [4]. The systole and diastole phases are the time intervals between S1 and S2, and between S2 and S1, respectively. For normal heart sounds, the heart valves produce low frequency transient signals. On the other hand, pathological heart sounds are called murmurs [1]. They are result of turbulence in blood flow through stenotic cardiac valves, or regurgitation through the atrioventricular valves. Heart murmurs produce high-frequency noise-like sounds [4]. In this study, we will focus on normal heart sounds and two cases of heart valve diseases, which are aortic stenosis and mitral insufficient.

\section{B. $\underline{\text { Heart Signals Simulation }}$}

Modeling and simulation in medical and health sciences play an important role to overcome many limitations of experimental studies on real animals or humans, e.g. financial limitations and ethical considerations [5]. Therefore, there is a growing interest in the use of simulations in medical education or training, and for accelerating advancements of diagnostic and therapeutic technologies.

Computer-based simulation of physiological signals, especially the signals resulted from the heart, has been successfully applied in many previous studies. Almost researchers focused on the modeling and analysis of the electrocardiogram (ECG) signals over several years [6-8]. In contrast, a few studies proposed models for representing the PCG signals $[9,10]$. However, the analysis of heart sounds and 
murmurs using DWT decompositions were widely investigated in the literature $[4,11$, 12]. Additionally, cardiac simulators based on a microcontroller present a low cost, portable, and practical tool to create standard generator of bio-signals for different health status of the heart. Paul et al. [13] developed an ECG simulator including bradycardia and tachycardia waves based on the ATmega32 Microcontroller. Using embedded dsPIC30F6012 microcontroller, Gergely et al. [14] proposed a digital resampling method to characterize the pathological PCGs.

The main aim of this work is to develop a graphical heart sounds generator with showing cardiac valve diseases. Compared to the previous studies, the developed heart sounds and murmurs generator adds the following contributions:

- No mathematical model of the heart sounds is required, because it generates typical PCGs based on real patient datasets

- Medical and engineering students can easily use it for studying different clinical scenarios of the heart status

- It can be used as an electronic tester for developed biomedical signal processing methods of automatic heart diseases diagnosis

The remainder of this paper is organized as follows. Section II gives a detailed description of each component of the developed heart sounds generator. Simulation results are presented and evaluated in Section III. Finally, Section VI concludes the microcontroller-based sounds generator of the heart as a potential useful tool in academia.

\section{Materials and Methods:}

\section{A. Materials}

Three sets of heart sounds and murmurs data were used in this study. The datasets are taken from a public database produced by the CliniSurf, Faculty of Medicin, University of Bern, Switzerland [15]. They contain 515 heart cycles with a sample rate of 44.1 $\mathrm{kHz}$.

The Arduino is a single-board microcontroller and a software suite for programming it [16]. The hardware consists of a simple open hardware design for the controller with an Atmel AVR processor and on-board I/O support. The basic Arduino connects to the computer using a standard USB cable, which provides both a serial connection to a PC and the $5.0 \mathrm{~V}$ power supply needed to operate. For the Arduino software, a standard programming language derived from the $\mathrm{C}++$ and the boot loader runs on the board. An Integrated Development Environment (IDE) contains a text-editor and compiler that translates the programmer's code into a binary hex file, which can be uploaded directly to the microcontroller. The features of Arduino ATmega 2560 microcontroller board have been employed to develop the heart sounds generator. It has 54 digital input/output 
pins, 16 analog inputs, 4 UART (hardware serial ports), a $16 \mathrm{MHz}$ ceramic resonator (oscillator), USB connection, a power jack, and a reset button, as depicted in Fig. 1.

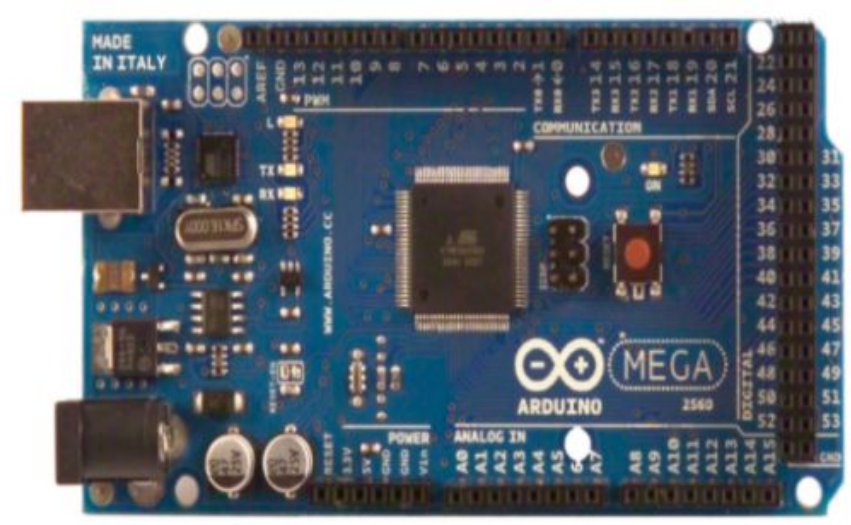

Figure (1): Arduino ATmega 2560 microcontroller board [16].

\section{B. Methods}

Discrete Wavelet Transform (DWT) is widely used in many signal processing applications such as object recognition, video compression, and numerical analysis [17]. The DWT can efficiently compute a compact representation of signals in multi timefrequency resolutions with only a few terms compared to the short time Fourier transform (STFT) [18]. The DWT of a signal is defined by Eq. 1, where $\psi(t)$ is called the mother wavelet, which is a time function with finite energy and fast decay; $j$ and $k$ are dilation (or scale) and translation indices respectively.

$$
W(j, k)=\sum_{j} \sum_{k} x(k) 2^{-\frac{j}{2}} \Psi\left(2^{-1} n-k\right)
$$

The DWT can be viewed as a constant filter bank with octave spacing between the centers of the filters such that the wavelet transform decomposes a discrete signal, $x[n]$, into two sub-signals of half its length. Each octave can be presented as a pair of finite impulse response (FIR) filters at least for one-dimensional (1D) signals [17]. One filter of the analysis DWT pair is a low pass filter (LPF), $g[n]$, and the other is a high pass filter (HPF), $h[n]$. The LPF produces the average or approximation sub-signal, $y_{\text {low }}[k]$, while the HPF produces the detail or fluctuation sub-signal, $y_{\text {high }}[k]$, after down sampling by 2 as defined in Eq. 2 and 3, respectively. 


$$
\begin{aligned}
& y_{\text {low }}[\mathbf{l k}]=\sum_{\mathbf{n}} \mathrm{x}[\mathrm{n}] \mathrm{g}[2 \mathrm{k}-\mathrm{n}] \\
& \mathbf{y}_{\mathbf{h i g h}}[\mathbf{k}]=\sum_{\mathbf{n}} \mathrm{x}[\mathbf{n}] \mathbf{h}[2 \mathrm{k}-\mathrm{n}]
\end{aligned}
$$

In the pyramidal algorithm of multiresolution analysis (MRA) using the DWT, the average signal feeds into another set of filters to produce the average and detail of the next octave. The outputs of each octave have only the half of input's data with a few coefficients of the filters. Therefore, the wavelet representation has approximately the same size of the original signal, as shown in Fig. 2.

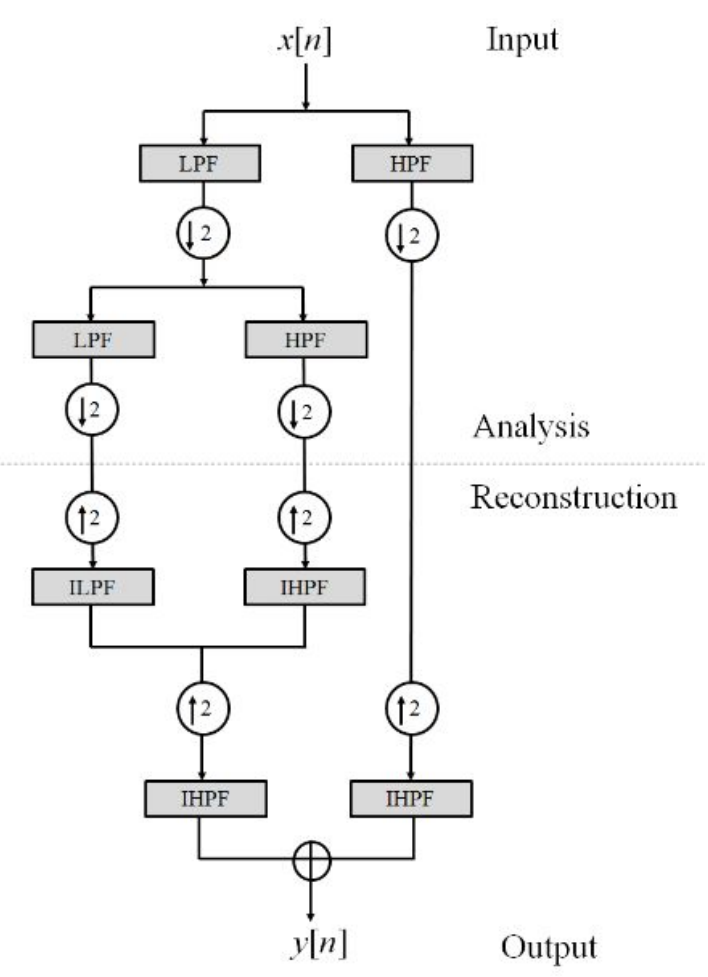

Figure (2): A two-step wavelet analysis and reconstruction.

The Daubechies four-coefficient (DUB4) wavelet [19] is used here to represent the heart sounds and murmurs, because it satisfies the orthogonality conditions, and has a

superior smoothing effect on signals [20]. Fig. 3 shows the three-level DUB4 wavelet decomposition for one cycle of normal heart sounds. The third level of approximation 
$\left(A_{3}\right)$ of the heart sounds was selected to prepare the tested datasets for saving in the memory of the microcontroller without losing the main features of original signals, as shown in Fig. 3(f). Furthermore, the approximation $A_{3}$ of heart signals is smooth and noise free because of the wavelet filtering.

\section{Results and Evaluation:}

The developed heart sounds and murmurs generator has been designed and implemented using two Arduino ATmega 2560 microcontroller boards, three 8-bits digital-to-analog converters (DACs), and a three position rotary switch to select a specific health status of the heart sounds as the displayed output signal. The Proteus design suite V8.2 (Labceneter Electronics Ltd., England) was used to simulate and test this hardware design, as shown in Fig. 4. The final results of the microcontroller-based heart sounds generator for the normal heart sounds, aortic stenosis, and mitral insufficient are depicted in Fig. 5.

In order to evaluate the output accuracy of the heart sounds generator, the root mean square error (RMSE) and maximum absolute error $\left(\mathrm{E}_{\max }\right)$ have been computed in Eq. 4 and 5, respectively, where $x_{O}$ and $x_{A}$ are the original and the reconstructed third level wavelet approximation of heart sounds for one heartbeat of each dataset with length $N=$ 35280 samples.

$$
\begin{aligned}
& \text { RMSE }=\left(\frac{1}{N} \sum_{i=1}^{N} \mathrm{~g}\left[\mathrm{x}_{0}-7 \mathrm{x}_{\mathrm{A}}\right]^{2}\right)^{\frac{1}{2}} \\
& \mathbf{E}_{\max } \max _{\mathbf{N}\left|\mathbf{x}_{0}-\mathbf{x}_{\mathrm{A}}\right|} \mid
\end{aligned}
$$

Table 1 illustrates the values of $E_{\max }$ and RMSE for all tested datasets of heart sounds and murmurs generator. The Daubechies wavelet is the most accurate type of wavelets for analyzing the PCG recordings [14]. Therefore, the results demonstrated that the filtered heart sounds using the DWT are almost equal to the original signals with RMSE of $10^{-10}$ and $\mathrm{E}_{\max }$ of $10^{-3}$ Volt. Consequently, the developed generator of heart sounds is clinically accepted, because it still contains the diagnostic information of the heart health status without any distortion.

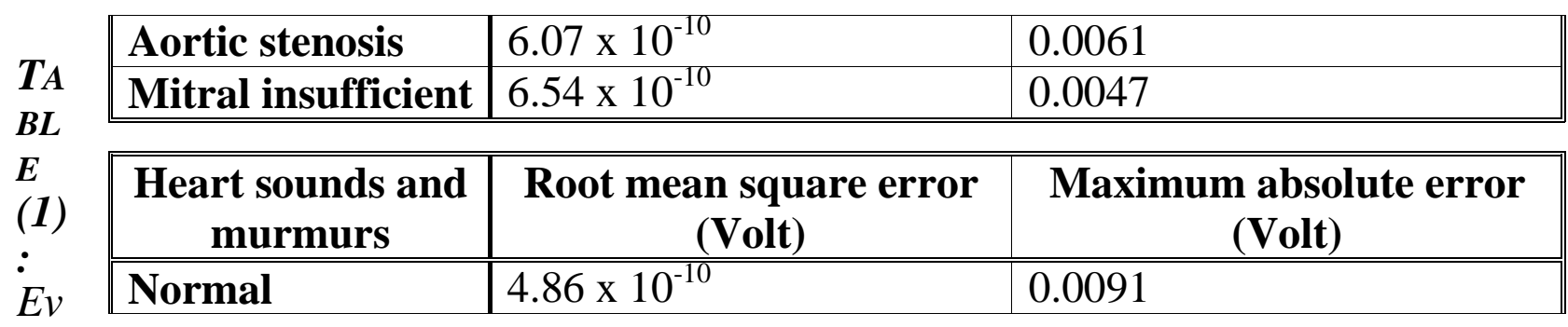



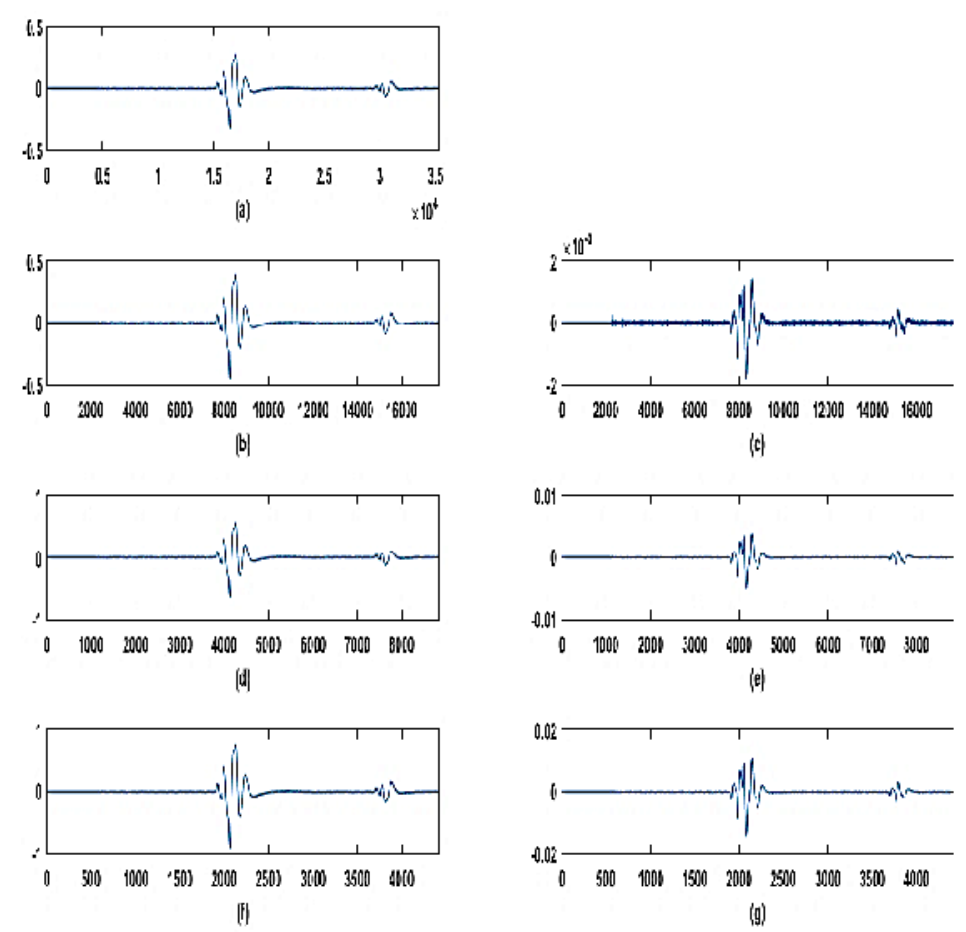

Figure (4): A three-level decomposition of the normal heart sounds signal using the DUB4 wavelet: (a) the original heart sounds signal, $(b-c)$ the first level of approximation $A_{1}$ and detail $D_{1},(d-e)$ the second level of approximation $A_{2}$ and detail $D_{2}$, and $(f-g)$ the third level of approximation $A_{3}$ and detail $D_{3}$. 


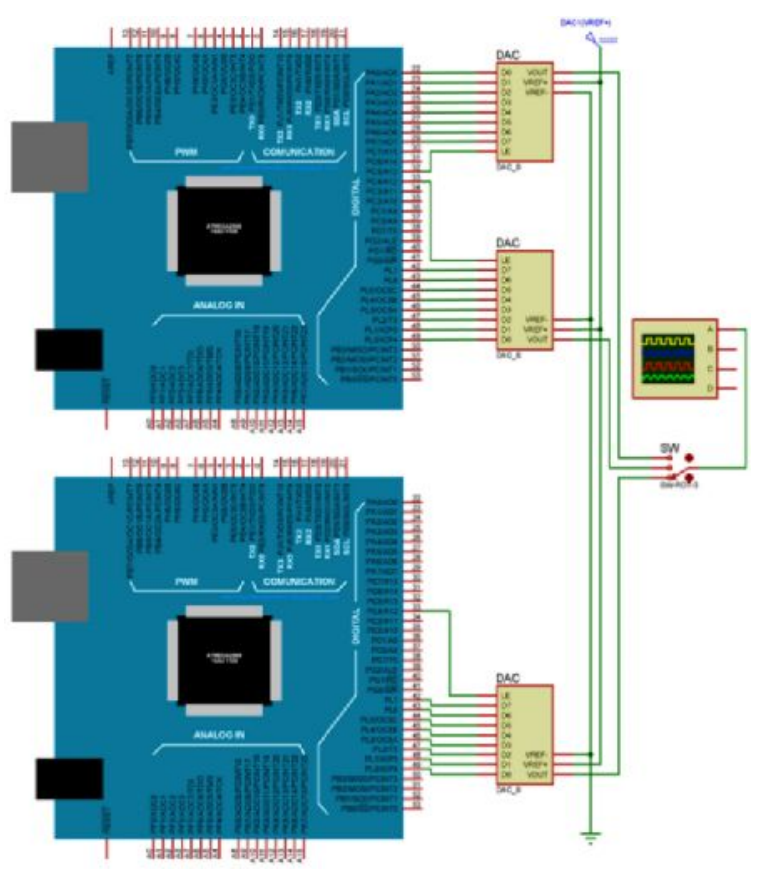

Figure (3): Schematic diagram of the develped heart sounds generator using the Arduino ATmega 2560 microcontroller board.

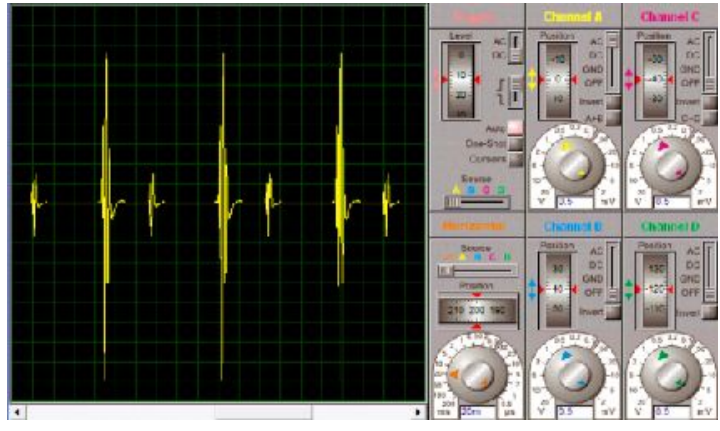

(a)

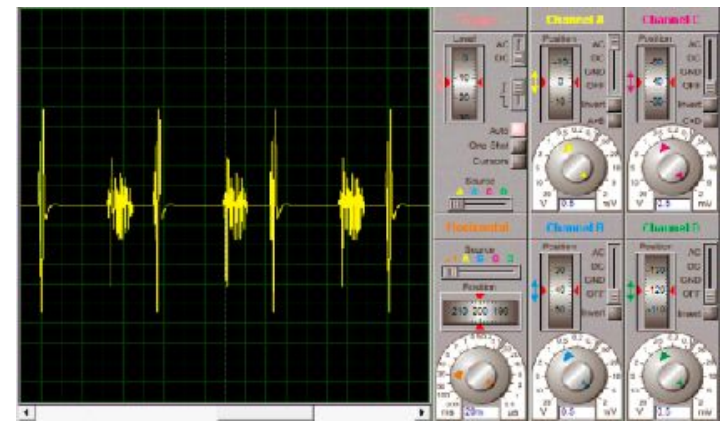

(b)

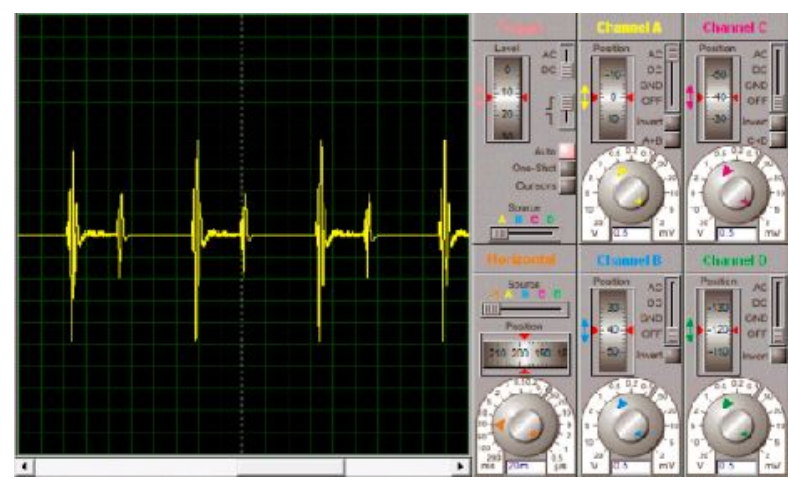

(c) 
Figure (5): Screenshots of three different heart sounds and murmurs as output signals of the microcontroller: (a) normal heart sounds, (b) aortic stenosis, and (c) mitral insufficient.

\section{Conclusions and Future Work:}

Generation of normal heart sounds and murmurs have been successfully implemented using Arduino ATmega 2560 microcontroller in this study. The developed heart sounds generator is cost effective and portable. It can be easily extended to include other diseased heart sounds with considering hardware specifications of the microcontroller, e.g. The memory size and limited number of digital and/or analog output ports.

Currently, the real hardware prototypes of our electronic heart sounds generator are under implementation to be used as a technical laboratory course for BSc students, and also for postgraduate studies of heart signals analysis in the field of biomedical engineering at Faculty of Electronic Engineering (FEE), Menoufia University, Egypt.

\section{References:}

[1] J. G. Webster, The Physiological Measurement Handbook: CRC Press, 2014.

[2] Z. Syed, D. Leeds, D. Curtis, F. Nesta, R. A. Levine, and J. Guttag, "A Framework for the Analysis of Acoustical Cardiac Signals," Biomedical Engineering, IEEE Transactions on, vol. 54, pp. 651-662, 2007.

[3] M. Brusco and H. Nazeran, "Development of an Intelligent PDA-based Wearable Digital Phonocardiograph," in Engineering in Medicine and Biology Society, 2005. IEEE-EMBS 2005. 27th Annual International Conference of the, 2005, pp. 3506-3509.

[4] F. Safara, S. Doraisamy, A. Azman, A. Jantan, and S. Ranga, "Wavelet Packet Entropy for Heart Murmurs Classification," Advances in Bioinformatics, vol. 2012, p. 6, 2012.

[5] J. A. Sokolowski and C. M. Banks, Modeling and Simulation in the Medical and Health Sciences: Wiley, 2012.

[6] M. I. Owis, A. H. Abou-Zied, A. B. M. Youssef, and Y. M. Kadah, "Study of features based on nonlinear dynamical modeling in ECG arrhythmia detection and classification," Biomedical Engineering, IEEE Transactions on, vol. 49, pp. 733-736, 2002. 
[7] P. E. McSharry, G. D. Clifford, L. Tarassenko, and L. A. Smith, "A dynamical model for generating synthetic electrocardiogram signals," Biomedical Engineering, IEEE Transactions on, vol. 50, pp. 289-294, 2003.

[8] E. K. Roonizi and R. Sassi, "A Signal Decomposition Model-Based Bayesian Framework for ECG Components Separation," Signal Processing, IEEE Transactions on, vol. 64, pp. 665-674, 2016.

[9] A. Almasi, M. B. Shamsollahi, and L. Senhadji, "A dynamical model for generating synthetic Phonocardiogram signals," in Engineering in Medicine and Biology Society, EMBC, 2011 Annual International Conference of the IEEE, 2011, pp. 5686-5689.

[10] M. Jabloun, P. Ravier, O. Buttelli, R. Lédée, R. Harba, and L.-D. Nguyen, "A generating model of realistic synthetic heart sounds for performance assessment of phonocardiogram processing algorithms," Biomedical Signal Processing and Control, vol. 8, pp. 455-465, 2013.

[11] S. Babaei and A. Geranmayeh, "Heart sound reproduction based on neural network classification of cardiac valve disorders using wavelet transforms of PCG signals," Computers in Biology and Medicine, vol. 39, pp. 8-15, 2009.

[12] S. Yuenyong, A. Nishihara, W. Kongprawechnon, and K. Tungpimolrut, "A framework for automatic heart sound analysis without segmentation," Biomedical engineering online, vol. 10, pp. 13-13, 2011.

[13] A. D. Paul, K. R. Urzoshi, R. S. Datta, A. Arsalan, and A. M. Azad, "Design and Development of Microcontroller Based ECG Simulator," in 5th Kuala Lumpur International Conference on Biomedical Engineering 2011. vol. 35, N. Osman, W. Abas, A. Wahab, and H.-N. Ting, Eds., ed: Springer Berlin Heidelberg, 2011, pp. 292-295.

[14] S. Gergely, M. N. Roman, and C. Fort, "Multirate Sampling in PCG Signal Correlation," in International Conference on Advancements of Medicine and Health Care through Technology. vol. 36, S. Vlad and R. Ciupa, Eds., ed: Springer Berlin Heidelberg, 2011, pp. 198-201.

[15] CliniSurf website. (2015). Available: http://elearning.studmed.unibe.ch/clinisurf/htmls/kardio_heartauscultation.html?clini surf|kardio|heartauscultation

[16] M. Böhmer, Beginning Android ADK with Arduino: Apress, 2012.

[17] M. Weeks, Digital Signal Processing Using MATLAB \& Wavelets: Jones \& Bartlett Learning, 2011.

[18] S. G. Mallat, "A theory for multiresolution signal decomposition: the wavelet representation," Pattern Analysis and Machine Intelligence, IEEE Transactions on, vol. 11, pp. 674-693, 1989. 
[19] I. Daubechies, "Orthonormal Bases of Compactly Supported Wavelets II. Variations on a Theme," SIAM Journal on Mathematical Analysis, vol. 24, pp. 499-519, 1993.

[20] A. Samant and H. Adeli, "Feature Extraction for Traffic Incident Detection Using Wavelet Transform and Linear Discriminant Analysis," ComputerAided Civil and Infrastructure Engineering, vol. 15, pp. 241-250, 2000. 